\title{
Towards better anion exchange membranes.
}

\author{
Zhengjin Yang, Tongwen $\mathrm{Xu}^{*}$ \\ Collaborative Innovation Center of Chemistry for Energy Materials, School of Chemistry and Material Science, \\ University of Science and Technology of China, China
}

Anion exchange membranes (AEMs) refer to polymers in the membrane shape, having positively charged groups (fixed groups) alongside the polymer chain, which have aroused intense scholar interest. AEMs serve a critical component in various electrochemical systems/devices [1-5]. Currently, increasing the hydroxide conductivity and enhancing the chemical stability of AEMs are the most challenging. Herein, I want to highlight endeavors in fabricating chemically stable and highly conductive AEMs to bring this discussion to a deeper level.

Constructing pendant type AEMs facilitates the formation of micro-phase separated morphology, offers more (or more efficient) hydroxide ion transport "channels" and leads to improved hydroxide conductivity. Micro-phase segregated morphology stems from the enhanced mobility of fixed groups via adding long spacers between the polymer backbone and the fixed groups. Strategies to implement such spacers may include lithiation reaction [6], Friedel-Crafts reaction [7], Suzuki coupling reaction [8] and the more straight-forward Menshutkin reaction [9]. Results suggest that by adding flexible spacers, enhanced phase segregated morphology forms, which consequently leads to increased hydroxide conductivity and suppressed water swelling. An interesting yet to be confirmed fact is that length of the spacers has a distinctive impact on the trend of how obvious an increase in hydroxide conductivity is. Currently, more researchers adopt the act of inducing phase segregated morphology in AEMs by adding flexible spacers. We should also keep in mind that the connection between the polymer backbone and the flexible head groups play an extremely important role in determining the chemical stability of the resulting AEMs [7], especially when an electronwithdrawing effect is concerned [10].

Creating nano-sized hydroxide ion transport channels by stopping the polymers from packing efficiently. By increasing the mobility of the functional groups, driven by the hydrophilicity/ hydrophobicity difference they will segregate and form ion transport channels. An extreme situation is to render the AEMs intrinsically micro-porous and all "pores" could serve as ion transport channels, with less resistance. Tröger's base polymers (TB polymers), because of their contorted chain structure, have intrinsic nano-sized pores [11]. We have demonstrated that an unprecedented hydroxide conductivity of $164.4 \mathrm{mS} / \mathrm{cm}$ can be attained at a relatively low ion-exchange capacity of 0.82 $\mathrm{mmol} / \mathrm{g}$. The results suggest that within the confined space, hydroxide ions exhibit distinctly different transport behaviors as opposed to that in bulk solutions. Similar observations were reported by Prof. Yamaguchi, who found that AEM from a trimethylbenzyl ammonium-appended PIM (polymers of 9 intrinsic micro-porosity) afforded $\mathrm{OH}$ - conductivity of $65 \mathrm{~m} / \mathrm{cm}$ as well as long-term durability [12].

Turing passive hydroxide transport into active transport. Rendering the "fixed" functional groups movable, we have developed AEMs containing mobile ion shuttles via host-guest interactions and disrupting this interaction imparts the side chains with enhanced mobility and increased hydrodynamic radius [13]. The thermally triggered ion shuttling effect endows the polyrotaxane hydroxide conductivity of $180 \mathrm{mS} / \mathrm{cm}$ at $90^{\circ} \mathrm{C}$, while one remaining challenge is to maintain high $\mathrm{OH}-$ conductivity by preventing the diffusion loss of the shuttles under harsh alkali conditions.

Noting that current chemical stability evaluations are exsitu and totally different from the actual working condition. In a practical AEMFC, the attack of hydroxide ions on AEMs occurs in the absence, or with scarce amount of water. Dekel et al. [14] proved that water molecules have a critical effect on the alkaline stability of quaternary ammonium cations and with increasing amount of water molecules solvating $\mathrm{OH}-$ ions, their nucleophilicity and basicity are decreased, leading to slower AEM degradation rate. Therefore, developing standard procedures/methods to make AEM stability assessment approaching the practical working specifications is urgent. Despite of the unresolved challenges, great achievements have been made in designing better AEMs, with both high conductivity and excellent chemical stability.

\section{Acknowledgement}

Financial supports from the National Natural Science Foundation of China (Nos. 21720102003) and International Partnership Program of Chinese Academy of Sciences (No. 21134ky5b20170010) are gratefully acknowledged.

\section{References}

1. Varcoe JR, Atanassov P, Dekel DR, et al. Anion-exchange membranes in electrochemical energy systems. Energy Environ Sci. 2014;7:3135.

2. Wang YJ, Qiao JL, Baker R, et al. Alkaline polymer electrolyte membranes for fuel cell applications. Chem Soc Rev. 2013;42:5768.

3. Hickner MA, Herring AM, Coughlin EB. Anion exchange membranes: Current status and moving forward. J Polym Sci B Polym Phys.2013;51:1727.

4. Soloveichik GL. Flow batteries: current status and trends. Chem. Rev. 2015;115:11533.

Mater Sci Nanotechnol 2017 Volume 1 Issue 1 
5. Couture A, Alaaeddine F, Boschet B. Polymeric materials as anion-exchange membranes for alkaline fuel cells. Prog. Polym. Sci. 2011;36:1521.

6. Dang HS, Weiber EA, Jannasch P. Poly(phenylene oxide) functionalized with quaternary ammonium groups via flexible alkyl spacers for high-performance anion exchange membranes. J Mater Chem. 2015;3:5280.

7. Hibbs MR. Alkaline stability of poly(phenylene)-based anion exchange membranes with various cations. Polym Phys. 2013;51:1736.

8. Yang Z, Zhou J, Wang S, et al. A strategy to construct alkalistable anion exchange membranes bearing ammonium groups via flexible spacers. J Mater Chem. 2015;3:15015.

9. Wang $\mathrm{J}, \mathrm{Gu} \mathrm{S}$, Xiong R, et al. Hyper-branched anion exchange membrane with high conductivity and chemical stability. ChemSusChem. 2015;8:4229.

10. Arges CG, Ramani V. Polystyrene-block-poly(ethylene-ran- butylene)-block-polystyrene triblock copolymer separators for a vanadium-cerium redox flow battery. Proc Natl Acad Sci. $2013 ; 110: 2490$.

11. Carta M, Malpass-Evans R, Croad M, et al. An efficient polymer molecular sieve for membrane gas separations. Science. 2013;339:303.

12. Ishiwari F, Sato T, Yamazaki H, et al. Rigid-to-flexible conformational transformation: An efficient route to ringopening of a tröger's base-containing ladder polymer. J Mater Chem. 2016;4:17655.

13. Ge X, He Y, Guiver MD, et al. Alkaline anion-exchange membranes containing mobile ion shuttles. Adv Mater. 2016;28:3467.

14. Dekel D, Amar RM, Willdorf S, et al. Effect of water on the stability of quaternary ammonium groups for anion exchange membrane fuel cell applications. Chem Mater. 2017;29:4425.

\section{*Correspondence to:}

Tongwen $\mathrm{Xu}$

School of Chemistry and Material Science

University of Science and Technology of China

P.R. China.

Tel: +86-551-63601587

E-mail: twxu@ustc.edu.cn 